\title{
Splenic abscess secondary to sleeve gastrectomy leak
}

\author{
Hiba Shanti, Firas Obeidat \\ Department of General Surgery, Jordan University Hospital, Amman, Urdun
}

\begin{abstract}
Sleeve gastrectomy is currently a stand-alone bariatric procedure with a low complication profile. A rare complication of leak following sleeve gastrectomy was reported in this study. A 34-year-old male patient with body mass index of 69.9 was admitted to hospital 4 months after sleeve gastrectomy with epigastric pain and fever, and leak at the gastroesophageal junction was observed on computed tomography scan. In addition, he had splenic vein thrombosis with infected partial splenic infarction with abscess formation. Endoscopic stent was used to cover the leak and percutaneous drainage of the splenic abscess was performed. Partial infarction of the spleen is not a rare occurrence after laparoscopic sleeve gastrectomy. However, abscess development is extremely rare. Familiarity with these rare complications will allow for prompt diagnosis and treatment.
\end{abstract}

Keywords: Bariatric surgery; gastrectomy leak; laparoscopic sleeve gastrectomy.

\section{Introduction}

Laparoscopic sleeve gastrectomy (LSG) is rapidly gaining momentum in bariatric surgery. LSG has the advantages of being a less complex procedure than other bariatric procedures with less malnutritive effect, comparable weight loss, and resolution of comorbidities to Roux-en-Y gastric bypass. Reported complications after LSG include staple line leak, bleeding, sleeve stricture, delayed gastric emptying, intra-abdominal abscess, splenic injury, and venous thrombosis. ${ }^{[1]}$ Formation of splenic abscess is an extremely rare consequence of LSG described in two case reports. ${ }^{[2]}$ Its rareness and nonspecific clinical presentation could make the diagnosis difficult and could be easily confused with leak and subdiaphragmatic abscess. The aim of this paper was to present a unique case of late gastric leak complicated by splenic abscess formation.

\section{Case Report}

A 34-year-old male, morbidly obese patient with a body mass index of 69.9 and weighing 202 kilograms was admitted to our clinic. His comorbidities included hypertension treated with Enalapril. He had no prior surgical history and underwent LSG. The immediate postoperative course was uneventful. The surgical technique involved complete mobilization of the stomach by dividing the greater omentum from the stomach using Ligasure ${ }^{\mathrm{TM}}$ (Covidien, USA), starting $2 \mathrm{~cm}$ from the pylorus and extending up to the gastroesophageal junction. A 38-Fr calibration bougie 


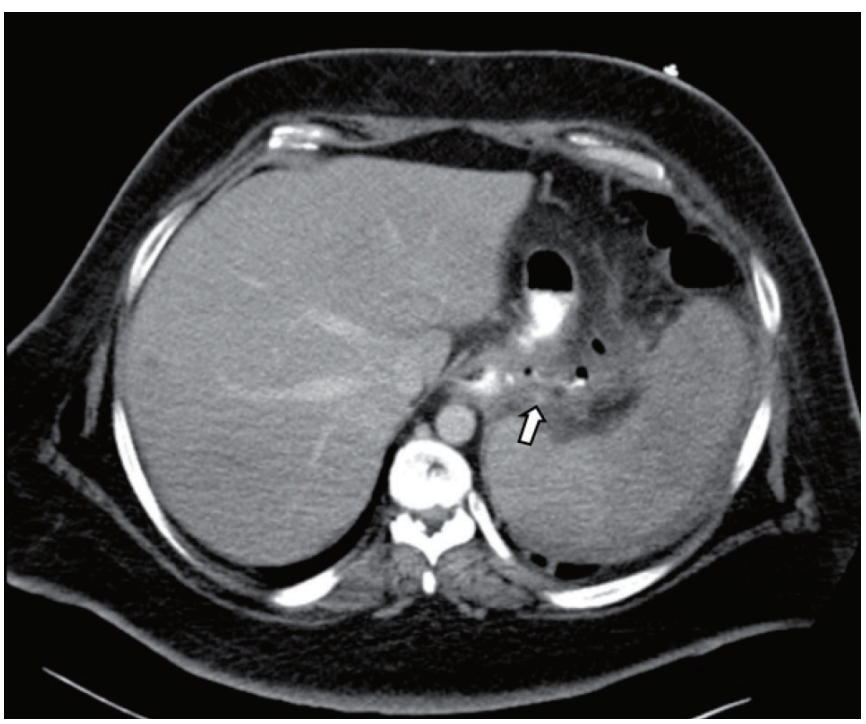

Figure 1. CT scan shows evidence of contrast leak at the gastroesophageal junction.

was inserted by the anesthesiologist along the lesser curvature of the stomach. The resection began with the use of a green Endo GIA stapler (Covidien, USA) starting $4 \mathrm{~cm}$ from the pylorus and continued toward the angle of His using a blue Endo GIA stapler (Covidien, USA). The staple line was reinforced using seromuscular invaginating V-Loc ${ }^{\mathrm{TM}}$ sutures (Covidien, USA).

The patient was discharged on postoperative second day after a negative upper GI study done routinely. The patient attended his first postoperative visit and subsequent three visits on the postoperative first, second and third months. All follow-ups were unremarkable except for occasional vomiting. He lost $39 \mathrm{~kg}$ after 3 months.

Four months after LSG, the patient presented with epigastric pain, leukocytosis, and a high grade fever for a duration of four days. A computed tomography (CT) scan revealed evidence of contrast leak at the gastroesophageal junction (GEJ) with no definite collection (Figure 1). In addition, splenic vein thrombosis was noted with evidence of splenic infarction involving more than $50 \%$ of the spleen (Figure 2). The patient was placed on antibiotics and therapeutic dose enoxaparin. He was kept on nothing orally and started on total parenteral nutrition (TPN). Follow-up CT scan, carried out one week later due to persistent leukocytosis and fever, showed a large splenic abscess (Figure 3). Percutaneous drain was inserted under CT guidance, which was successful in the resolution of the abscess as demonstrated by a new CT scan performed after two weeks. The patient showed clinical improvement with normalization of white cell count and

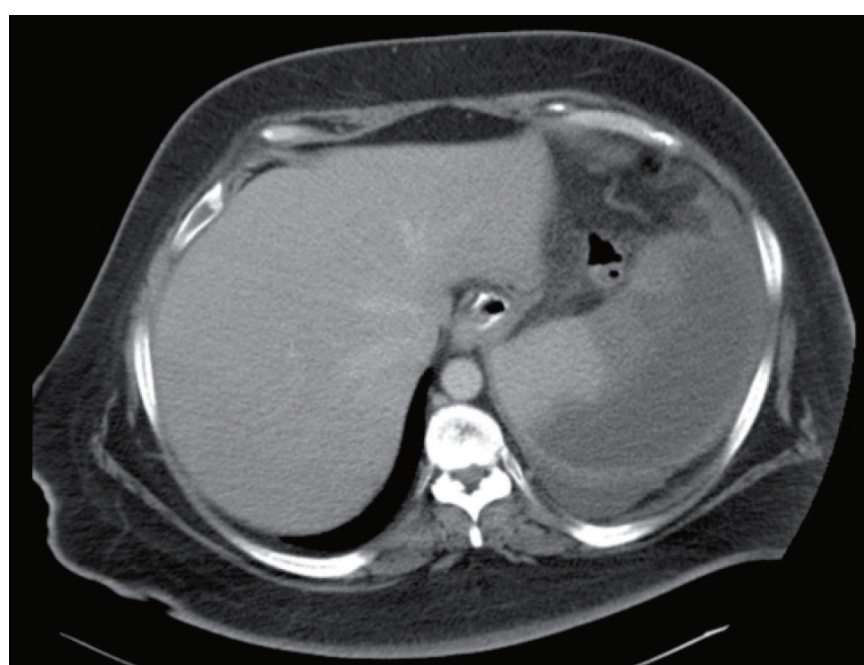

Figure 2. CT scan showing evidence of splenic infarction complicated by abscess formation (star).

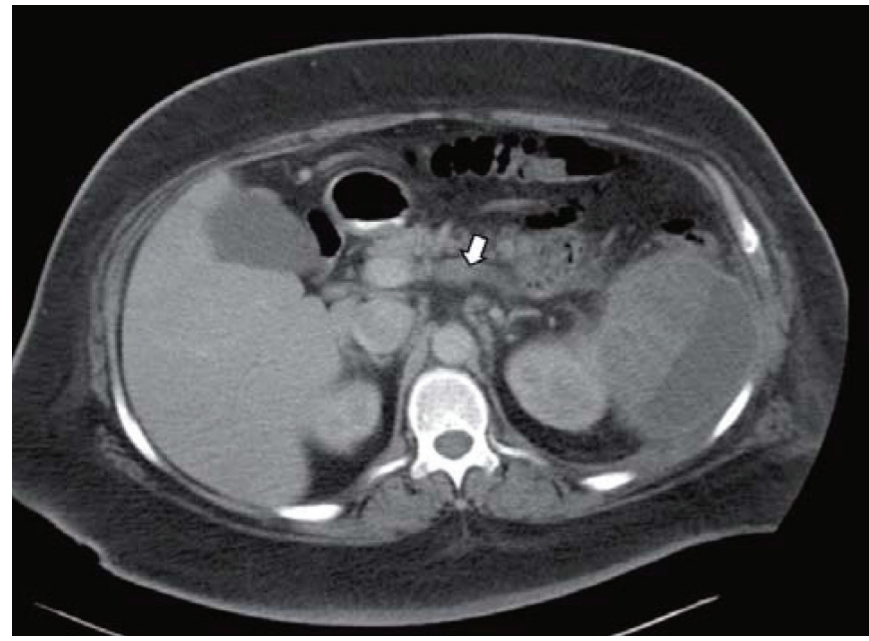

Figure 3. CT scan showing splenic vein thrombosis (arrow).

lack of fever. A covered metallic stent was used to cover the leak and was removed four weeks later. Upon removal, the site of leak revealed complete closure of the defect. The patient attended two subsequent visits and he was tolerating oral diet.

On his most-recent follow-up visit, he had a total 6-month weight loss of $68 \mathrm{~kg}$ (body mass index $46.4 \mathrm{~kg} / \mathrm{m}^{2}$ ) and excess weight loss of $52 \%$.

\section{Discussion}

LSG has become one of the most common bariatric procedures performed nowadays. Its simplicity and low risk profile make it more appealing. Leak at the lengthy stapler line is the most dreaded complication after LSG. Leak is reported to occur at a rate around $2.4 \%$; in a range 
of $0-8.9 \% \cdot{ }^{[1]}$ Most leaks occur at the proximal portion of the staple-line with $89 \%$ being at the GE junction (GEJ). ${ }^{[1]}$ Leak after LSG is multifactorial; relative ischemia and increased wall tension mostly at the GEJ play important roles. Other suggested factors may be related to the use of smaller bougie size, which can be attributed to the greater clearance at the GEJ given by a larger bougie size. ${ }^{[1]}$ BMI $>50$ also appears to be associated with a higher leak rate. ${ }^{[4]}$ Lastly, over-sewing of the stapler line does not show any decrease in the leak rate, while buttressing does. ${ }^{[5]}$

The diagnosis of staple line leak can be made based on clinical evidence with tachycardia being the cardinal sign. Other signs include fever, epigastric pain, leukocytosis, and elevated C-reactive protein. Leak can also be detected by extravasation of contrast in a swallow study or CT scan.

Leak treatment after LSG varies and depends on the extent of disruption, the extent of abdominal contamination, the site of the leak, and the time of diagnosis. Csendes classified leaks according to the time of diagnosis, including those detected before the third postoperative day (POD), those detected between fourth and seventh PODs, and those occurring after seventh POD $(\mathrm{POD}+8) \cdot{ }^{[3]}$ Our case demonstrated a rare, delayed presentation of leak after LSG. Avoiding surgical intervention after fourth POD is generally agreed upon. Operative management may be useful for debridement and drainage; however, primary closure is usually heralded by poor tissues and inflammation. ${ }^{[6]}$ Endoscopic stents have emerged for the management of gastric leaks but are only amenable for leaks at the proximal and mid-aspects of the sleeve. In addition, adequate drainage of any abdominal collection is of utmost importance. ${ }^{[7]}$ Parenteral nutrition or jejunostomy feeding is important for nutritional support, while oral feeding is withheld.

Aside from leaks, some rare complications have been reported following LSG. One of these is portomesenteric venous thrombosis. The cause of portomesenteric thrombosis is multifactorial; obesity itself is a well-known risk factor for venous thrombosis. Undiagnosed thrombophilia is another factor. Loco-regional factors predisposing to portomesenteric thrombosis include local inflammatory response and direct trauma or manipulation of the portomesenteric structures, which leads to endothelial injury and thrombus formation. Insufflation of the abdomen during laparoscopic procedures with subsequent increased intraabdominal pressure decreases mesenteric and portal venous flow. ${ }^{[8]}$
The clinical presentation of portomesenteric venous thrombosis is variable, consisting nonspecific abdominal pain, nausea, vomiting, diarrhea, and fever. In addition, peritoneal signs or shock due to bowel ischemia may occur and if the splenic vein is involved, splenic infarction may be seen. ${ }^{[8]}$ Occlusion of the main splenic artery or vein seldom produces splenic infarction. However, the ultimate branches are end-vessels and when any of these gets occluded, an area of infarction will occur immediately distal to the involved branch. This can be seen after the division of the short gastric arteries in gastric surgery. [9] Our patient had his short gastric vessels divided with postoperative splenic vein thrombosis; two factors ended up with splenic infarction.

Postoperative splenic infarction is usually silent and most cases will demonstrate resolution without clinical sequelae. ${ }^{[10]}$ The frequency of splenic infarction after LSG varies in the literature. In a study of two hundred and sixty-one patients undergoing LSG, Menenakos has reported one patient developing splenic infarction. ${ }^{[1]}$ In another study of two hundred and eighty-seven patients, the frequency of splenic infarction has been reported to be about 4.1\%. However, only $0.4 \%$ were symptomatic. ${ }^{[12]}$ When symptomatic, it often presents with vague or nonspecific signs and symptoms, usually including LUQ pain, fever, nausea and vomiting, and leukocytosis and may be difficult to differentiate from leak. An infarcted splenic area may progress into a subcapsular hematoma, get infected and progress into an abscess, or may even rupture into the abdomen. ${ }^{[13]}$ Larger volumes of infarction lead to greater rates of complications. To our knowledge, only two cases of splenic abscess following LSG have been reported and attributed to splenic infarct complications. ${ }^{[2]}$

Splenic abscess is an uncommon and life-threatening condition with an incidence ranging from 0.1 to $0.7 \%$ in various autopsy series. ${ }^{[14]}$ Splenic abscess is encountered in the presence of infection, trauma, emboli, or immunosuppression. It can result from hematogenous or contiguous bacterial spread from an intraabdominal infection. Splenic abscess can also occur in a previously infarcted area by direct seeding of the spleen from an adjacent infectious process. ${ }^{[13]}$ It is our belief that the large splenic infarction in relation to the nearby contamination from the gastric leak predisposed to splenic abscess in our patient.

The management of splenic abscess remains controversial. Splenectomy and antibiotics have generally been the 
definitive treatment. However, splenic preserving percutaneous strategies are more commonly used. ${ }^{[15]}$ These are most apt to unilocular abscesses and for the critically ill patient. Percutaneous drainage was successful in abscess resolution as demonstrated by a follow-up CT performed two weeks later.

\section{Conclusion}

While, most challenging complications can occur after LSG, the most dreaded complication is leakage. However, familiarity with the rare complications as portomesenteric thrombosis and splenic abscess will allow for a prompt diagnosis and treatment. Endoscopic stents and percutaneous drainage are safe and effective alternatives to surgery in the management of LSG complications.

\section{Disclosures}

Peer-review: Externally peer-reviewed.

Conflict of Interest: None declared.

\section{References}

1. Aurora AR, Khaitan L, Saber AA. Sleeve gastrectomy and the risk of leak: a systematic analysis of 4,888 patients. Surg Endosc 2012;26:1509-15. [CrossRef]

2. Gupta S, Singh O, Hastir A, Shukla S, Mathur RK. Splenic abscesses: Reports of two cases with review of the literature. Ann Trop Med Public Health 2012;5:273-7. [CrossRef]

3. Csendes A, Burdiles P, Burgos AM, Maluenda F, Diaz JC. Conservative management of anastomotic leaks after 557 open gastric bypasses. Obes Surg 2005;15:1252-6. [CrossRef]

4. Srinivasa S, Hill LS, Sammour T, Hill AG, Babor R, Rahman H. Early and mid-term outcomes of single-stage laparoscopic sleeve gastrectomy. Obes Surg 2010;20:1484-90. [CrossRef]

5. Choi YY, Bae J, Hur KY, Choi D, Kim YJ. Reinforcing the staple line during laparoscopic sleeve gastrectomy: does it have advantages? A meta-analysis. Obes Surg 2012;22:1206-13.

6. Oshiro T, Kasama K, Umezawa A, Kanehira E, Kurokawa Y. Successful management of refractory staple line leakage at the esophagogastric junction after a sleeve gastrectomy using the HANAROSTENT. Obes Surg 2010;20:530-4. [CrossRef]

7. Nguyen NT, Nguyen XM, Dholakia C. The use of endoscopic stent in management of leaks after sleeve gastrectomy. Obes Surg 2010;20:1289-92. [CrossRef]

8. Goitein D, Matter I, Raziel A, Keidar A, Hazzan D, Rimon U, et al. Portomesenteric thrombosis following laparoscopic bariatric surgery: incidence, patterns of clinical presentation, and etiology in a bariatric patient population. JAMA Surg 2013;148:340-6. [CrossRef]

9. Martínez DG, Sánchez AW, García AP. Splenic abscess after laparoscopic Nissen fundoplication: a consequence of short gastric vessel division. Surg Laparosc Endosc Percutan Tech 2008; 18:82-5. [CrossRef]

10. Salvi PF, Stagnitti F, Mongardini M, Schillaci F, Stagnitti A, Chirletti $P$. Splenic infarction, rare cause of acute abdomen, only seldom requires splenectomy. Case report and literature review. Ann Ital Chir 2007;78:529-32.

11. Menenakos E, Stamou KM, Albanopoulos K, Papailiou J, Theodorou D, Leandros E. Laparoscopic sleeve gastrectomy performed with intent to treat morbid obesity: a prospective single-center study of 261 patients with a median follow-up of 1 year. Obes Surg 2010;20:276-82. [CrossRef]

12. Stamou KM, Menenakos E, Gomatos IP, Panousopoulos SG, Smparounis S, Leandros $E$, et al. Clinical implications of sleeve gastrectomy as a source of spleen infarction or ischemia. Obes Surg 2011;21:1490-3. [CrossRef]

13. Arslan F, Batirel A, Tabak F, Mert A. Splenic abscess caused by MRSA developing in an infarcted area: case report and literature review. J Infect Chemother 2011;17:851-4. [CrossRef]

14. Ooi LL, Leong SS. Splenic abscesses from 1987 to 1995. Am J Surg 1997;174:87-93. [CrossRef]

15. Zerem E, Bergsland J. Ultrasound guided percutaneous treatment for splenic abscesses: the significance in treatment of critically ill patients. World J Gastroenterol 2006;12:7341-5. 